\title{
PI3-kinase-dependent activation of apoptotic machinery oc- curs on commitment of epidermal keratinocytes to terminal differentiation
}

Sam M Janes ${ }^{1}$, Tyler A Ofstad ${ }^{2}$, Douglas H Campbell ${ }^{3}$, Ayad Eddaoudi ${ }^{4}$, Gary Warnes ${ }^{5}$, Derek Davies ${ }^{6}$, Fiona M Watt $^{\top}$

${ }^{1}$ Centre for Respiratory Research, Rayne Institute, University College London, 5 University Street, London WC1E 6BT, UK; ${ }^{2}$ Graduate Program in Neurosciences, University of California, San Diego, CA 92093-0662, USA; ${ }^{3}$ Immune System Therapeutics Ltd, 235 Jones Street, Ultimo, NSW 2007, Australia; ${ }^{4}$ Molecular Immunology Unit, Institute of Child Health, University College London, 30 Guilford Street, London WC1N 1EH, UK; ${ }^{5}$ Institute of Cell and Molecular Science, Barts and The London School of Medicine and Dentistry, 4 Newark Street, London E1 2AT, UK; ${ }^{6}$ Cancer Research UK London Research Institute, 44 Lincoln's Inn Fields, London WC2A 3PX, UK; ${ }^{7}$ Cancer Research UK Cambridge Research Institute, Li Ka Shing Centre, Robinson Way, Cambridge CB2 ORE, UK

We have investigated the earliest events in commitment of human epidermal keratinocytes to terminal differentiation. Phosphorylated Akt and caspase activation were detected in cells exiting the basal layer of the epidermis. Activation of Akt by retroviral transduction of primary cultures of human keratinocytes resulted in an increase in abortive clones founded by transit amplifying cells, while inhibition of the upstream kinase, PI3-kinase, inhibited suspension-induced terminal differentiation. Caspase inhibition also blocked differentiation, the primary mediator being caspase 8. Caspase activation was initiated by $2 \mathrm{~h}$ in suspension, preceding the onset of expression of the terminal differentiation marker involucrin by several hours. Incubation of suspended cells with fibronectin or inhibition of PI3-kinase prevented caspase induction. At $2 \mathrm{~h}$ in suspension, keratinocytes that had become committed to terminal differentiation had increased side scatter, were 7-aminoactinomycin D (7-AAD) positive and annexin V negative; they exhibited loss of mitochondrial membrane potential and increased cardiolipin oxidation, but with no increase in reactive oxygen species. These properties indicate that the onset of terminal differentiation, while regulated by PI3-kinase and caspases, is not a classical apoptotic process.

Keywords: epidermis, integrins, extracellular matrix

Cell Research (2009) 19:328-339. doi: 10.1038/cr.2008.281; published online 2 September 2008

\section{Introduction}

The human interfollicular epidermis comprises multiple layers of keratinocytes. The basal layer is attached to an underlying basement membrane and contains at least two types of proliferating cells, known as stem and transit amplifying cells [1]. Stem cells have unlimited selfrenewal capacity and also give rise to cells that undergo

Correspondence: Fiona M Watt

Tel: +44-1223-404400; Fax: +44-1223-404573

E-mail: fiona.watt@cancer.org.uk

Received 30 May 2008; revised 17 July 2008; accepted 18 July 2008; published online 2 September 2008 terminal differentiation. Prior to differentiation the cells may divide a small number of times; such cells, with low self-renewal ability and high differentiation probability, are the transit amplifying cells. Once a cell has withdrawn irreversibly from the cell cycle and initiated terminal differentiation it moves out of the basal layer. Over the next few days or weeks it undergoes a well-defined series of changes in gene expression and cell morphology as it moves through first the spinous layer and then the granular layer towards the surface of the tissue. The cells in the final stages of terminal differentiation (cornified layer) are anucleate and have assembled transglutaminase cross-linked proteins and lipids into a structure known as the cornified envelope, which is essential for 
the barrier properties of the skin.

Keratinocytes can be isolated from human epidermis and cultured under conditions in which stem cells are preserved, as evidenced by the clinical applications of cultured epidermis as autologous grafts for burns victims [2]. The cultures therefore provide a useful experimental model with which to study how differentiation is controlled in an adult self-renewing epithelium. Clonal growth assays have been used both to identify cell surface markers that enrich for stem cells and to examine the genes that regulate exit from the stem cell compartment $[1,2]$. The putative stem cells found large clones that are a mixture of self-renewing cells and cells that initiate terminal differentiation. In contrast, transit amplifying cells form abortive clones that typically comprise fewer than 40 cells by 14 days in culture, all of which have initiated terminal differentiation and express the spinous layer marker, involucrin $[1,2]$.

One of the conditions that triggers human keratinocytes to undergo terminal differentiation is to place them as a single cell suspension in semi-solid medium [3,4]. Irreversible commitment of both stem and transit amplifying cells to terminal differentiation occurs within the first $4 \mathrm{~h}$; at this stage the cells have not started to express involucrin, but have withdrawn irreversibly from the cell cycle [4]. Expression of spinous layer markers, including involucrin and cornifin, begins by $10 \mathrm{~h}$, and mature cornified envelopes, representing the final stage of terminal differentiation, are assembled within $48 \mathrm{~h}$ [3-5]. The differentiation signal is mediated by loss of extracellular matrix (ECM) attachment and can be inhibited by ligating $\beta 1$ integrins with anti-integrin antibodies, fibronectin or a combination of ECM proteins $[4,6]$.

Keratinocyte terminal differentiation shares a number of features with apoptosis, including permanent withdrawal from the cell cycle, activation of caspases and transglutaminases, and stimulation by c-Myc $[5,7]$. Unligated $\beta 1$ integrins stimulate both apoptosis [8] and terminal differentiation $[9,10]$. There are, however, notable differences, such as the nuclear swelling, as opposed to condensation, that occurs in differentiating keratinocytes [5]. Furthermore, the kinetics of each process are distinct, with apoptosis occurring within minutes or hours and terminal differentiation taking place over the course of several weeks in vivo.

There is evidence that activation of Akt is involved in the initiation of keratinocyte terminal differentiation. While there is one report that inhibition of PI3K stimulates differentiation [11], most available data indicate that Akt activation plays a positive role in the process [1214]. Mice lacking both Akt1 and Akt2 die shortly after birth and have thin epidermis, with a reduced number of proliferating cells and reduced number of differentiating cell layers [15]. Conversely, transgenic activation of Akt in the epidermal basal layer stimulates proliferation and results in thickening of the interfollicular epidermis [16]. Akt is one of the genes that is upregulated in response to activation of the forkhead transcription factor FOXN1 [17]. In reconstituted human epidermis FOXN1 promotes the early stages of terminal differentiation (spinous layers), whereas Akt activation induces the late stages, including accumulation of cornified layers [17]. Pharmacological inhibition of PI3K blocks suspension-induced terminal differentiation, whereas inhibition of Akt does not [17], suggesting that Akt is not the only route by which PI3K exerts its effects.

There is also evidence that Akt protects keratinocytes from apoptosis. Activation of Akt in tumour-derived keratinocytes is negatively regulated by caspases and prevents cells from undergoing suspension-induced apoptosis [18]. This is in good agreement with the finding that inhibition of PI3K leads to death of cells that would otherwise have completed terminal differentiation [13, 14]. Knockdown of Akt1 leads to apoptosis in the suprabasal layers of in vitro reconstituted human epidermis and is alleviated by caspase inhibition [14]. Furthermore, $\mathrm{Bcl}-\mathrm{x}_{\mathrm{L}}$ and Akt cooperate to protect epidermal keratinocytes from UVB-induced apoptosis in mouse epidermis [19]. Taken together, these findings suggest that Akt is involved both as a survival factor and in initiation of terminal differentiation.

In the present report we have investigated the roles of the PI3-kinase/Akt pathway and caspases in commitment of keratinocytes to terminal differentiation. We show that committed keratinocytes have characteristics that are distinct from apoptotic cells, and we define features that will facilitate isolation of these cells for further analysis.

\section{Results}

Akt is activated on commitment to terminal differentiation in human epidermis

To examine the sites of Akt activation in vivo, we performed immunofluorescence staining of sections of human skin with antibodies to phospho-Akt ${ }^{473}$ (Figure $1 \mathrm{~A}$ and $1 \mathrm{C})$. Sections were also labeled with antibodies to E-cadherin (Figure 1B and 1C), which is localized to cell-cell borders in all the viable epidermal layers, and with Hoechst dye, which labels nuclei (Figure 1C). There was intense phospho-Akt immunoreactivity in the first 2-3 suprabasal layers. Phospho-Akt labeling declined in the upper spinous layers and was undetectable in the granular and cornified layers. Most cells in the epidermal basal layer did not label with the phospho-Akt antibody. 

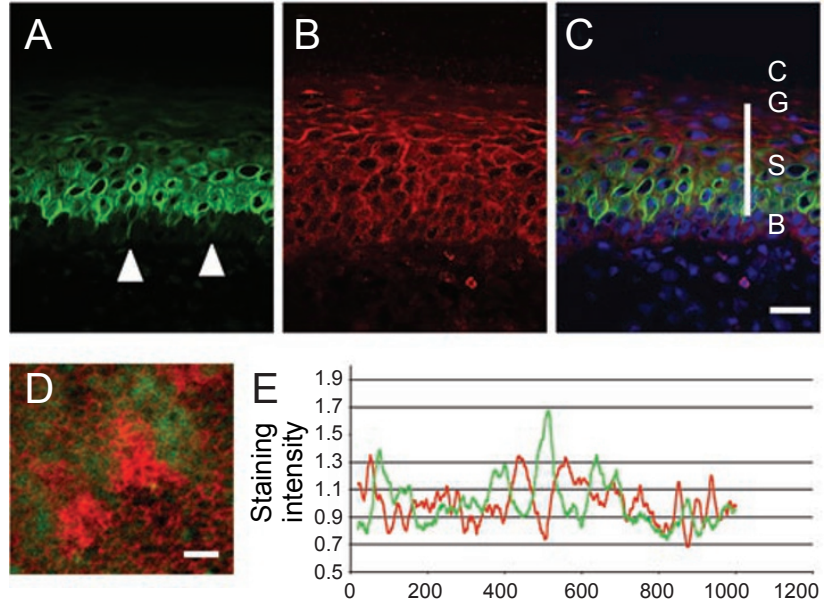

Figure 1 Phospho-Akt ${ }^{473}$ labeling of human epidermis. (A-C) Double immunofluorescence labeling with anti-phosphoserine $\mathrm{Akt}^{473}$ (green) and anti-E-cadherin (red), with Hoechst nuclear counterstain (blue). (A-C) show the same section. Arrowheads in (A) mark epidermal-dermal junction. (C) B: basal; S: spinous; G: granular; C: cornified layers. The white vertical line indicates viable suprabasal layers that express E-cadherin. (D) Basal layer of human epidermal whole mount labeled with anti-phospho $\mathrm{Akt}^{473}$ (green) and anti- $\beta 1$ integrin (red). The putative stem cells lie in the integrin-bright clusters; phospho Akt-labeled cells are absent from those clusters. (E) To determine the relative labeling intensity of $\beta 1$ integrins and phospho Akt in an epidermal wholemount, a line was drawn across a microscopical field and the red and green fluorescence intensities ( $y$-axis, arbitrary units) per unit length ( $x$-axis; each division is $200 \mu \mathrm{m}$ ) were determined [21, 49]. Scale bars: $10 \mu \mathrm{m}$ (A-C), $50 \mu \mathrm{m}$ (D).

However, individual cells with high levels of phosphoAkt extended processes into the basal layer, their shape suggesting that they were migrating into the suprabasal layers $[20,21]$.

Within the basal layer of human, but not mouse, interfollicular epidermis the putative stem cells are clustered and express high levels of $\beta 1$ integrins [22, 23]. This can be readily visualized by confocal microscopy of epidermal whole mounts, in which the basal layer of human interfollicular epidermis is viewed as an intact sheet [21]. When human epidermal whole mounts were double labeled with antibodies to phospho-Akt ${ }^{473}$ (green) and $\beta 1$ integrins (red) (Figure 1D) there was little co-expression. By quantitating the fluorescence signal along a straight line placed across a whole mount the inverse correlation between phospho-Akt and integrin levels is clearly seen: the peaks of red fluorescence do not coincide with the peaks of green fluorescence (Figure 1E).

Akt activation drives keratinocytes into the transit amplifying compartment in vitro
The localization of phospho-Akt ${ }^{473}$-positive cells suggested that Akt is phosphorylated in cells that are committed to terminal differentiation. To determine whether Akt stimulates differentiation in vitro, we introduced an activatable form of Akt into primary human keratinocytes via retroviral infection $[17,18]$. myrAktER is a membrane-targeted Akt construct in which Akt activation is dependent on 4-hydroxy-tamoxifen (4-OHT) [24]. An inactive version, A2AktER, was used as a control [24]. Keratinocytes were transduced with the Akt constructs and expression confirmed by probing the blots with an anti-ER antibody (data not shown; $[17,18]$ ). We have previously shown that there is a strong increase in $\mathrm{Akt}^{473}$ phosphorylation in keratinocytes transduced with myrAktER, while a slight increase in A2AktER-transduced cells indicates some leakiness of the construct [17].

Transduced keratinocytes were seeded at clonal density on a feeder layer and cultured in the presence or absence of $100 \mathrm{nM} 4-\mathrm{OHT}$ for 14 days. We then fixed and stained the dishes and scored the total number of colonies per dish and the proportion of those colonies that were abortive, that is, small colonies of differentiated cells attributable to transit amplifying cell founders [22]. We did not attempt to evaluate the sizes of non-abortive colonies, founded by putative stem cells [1]. 4-OHT treatment of cells expressing the control construct, A2AktER,
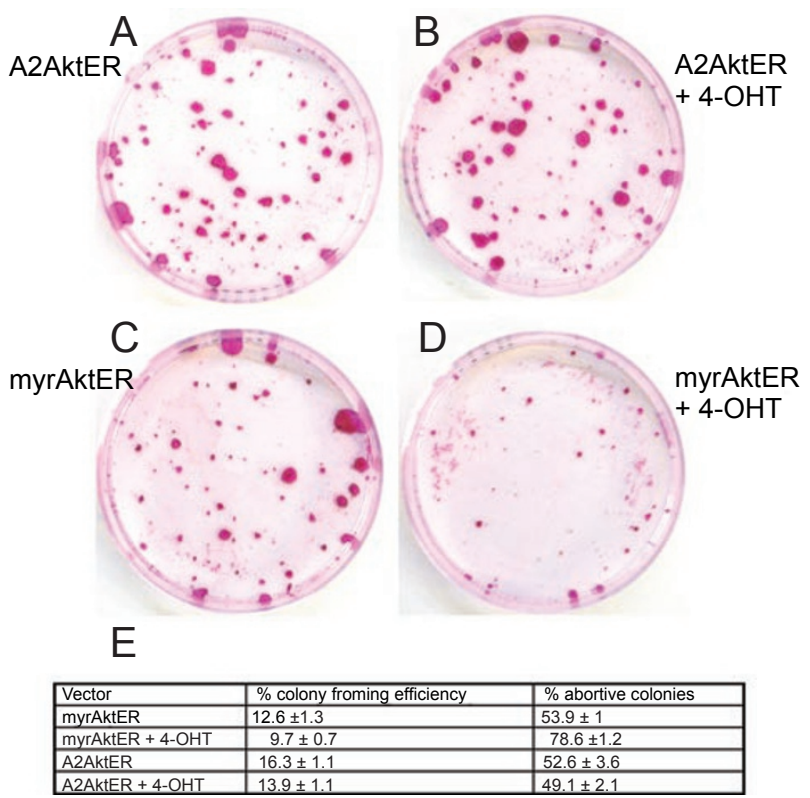

Figure 2 Effect of Akt activation on clonal growth of keratinocytes. Cells were transduced with $\operatorname{A2AktER~}(\mathbf{A}, \mathbf{B})$ or myrAktER (C, D) and treated with $100 \mathrm{nM}$ 4-OHT (B, D) or ethanol (vehicle control; A, C). (E) Quantitation of \% colony forming efficiency and $\%$ abortive colonies. 
did not affect colony formation (Figure 2A, 2B and 2E). In contrast, there was a statistically significant increase in abortive colonies in 4-OHT-treated cells transduced with myrAktER $(P<0.05$; Figure $2 \mathrm{C}-2 \mathrm{E})$. We conclude that Akt activation stimulates keratinocytes to move from the stem to the transit amplifying compartment.

Inhibition of PI3-kinase or caspases, but not p38MAPK or MEK1, blocks suspension-induced differentiation

When keratinocytes are disaggregated and placed in suspension, the majority of cells, whether stem cells or transit amplifying cells [22], initiate terminal differentiation and express spinous layer markers such as involucrin and cornifin. Within $4 \mathrm{~h}$ cells withdraw irreversibly from the cell cycle and will not proliferate if replated in culture; these cells are said to be committed to terminal differentiation because they have not yet begun to express involucrin. Overt terminal differentiation, as indicated by involucrin expression, begins at approximately $8 \mathrm{~h}$ in suspension [4].

Suspension-induced differentiation can be partially inhibited by ligating surface $\beta 1$ integrins with anti-integrin antibodies or with fibronectin $[4,6]$. If Akt activation stimulates keratinocytes to exit the stem cell compartment, a PI3-kinase inhibitor would be expected to block suspension-induced differentiation [17]. We assessed this by measuring induction of involucrin and cornifin, and down-regulation of $\beta 1$ integrins (Figure 3A-3E). Primary human keratinocytes were suspended in the presence of the PI3K inhibitor, LY294002 $(50 \mu \mathrm{M})$, fibronectin (100 $\mu \mathrm{g} / \mathrm{ml}$ ) or DMSO (diluent control). LY294002 inhibited differentiation in all three assays, inhibiting the induction of involucrin (Figure 3A) and cornifin (Figure 3B) and blocking $\beta 1$ integrin down-regulation (Figure 3C3E). The effect of LY294002 on induction of involucrin expression was much stronger than the effect of fibronectin (Figure 3A). As reported previously, control keratinocytes did not undergo suspension-induced apoptosis [5, 18]; furthermore, treatment with LY294002 did not stimulate apoptosis in suspension (data not shown).

Given the similarities between apoptosis and keratinocyte terminal differentiation, we examined whether caspase activation was required for suspension-induced differentiation. The broad-spectrum caspase inhibitor, $\mathrm{z}$-VAD-fmk $(100 \mu \mathrm{M})$, was as effective as LY294002 in preventing the induction of involucrin (Figure $3 \mathrm{~F}$ ) and cornifin (data not shown) expression and in blocking the down-regulation of $\beta 1$ integrins (Figure 3H-3J).

Caspase signalling can be broadly grouped into two pathways, the caspase 8-dependent extrinsic pathway, initiated by activation of death-domain-containing receptors $[25,26]$, and the caspase 9-dependent intrinsic pathway, typically activated by cytotoxic drugs or DNA damage. To examine the role of these pathways in keratinocyte suspension-induced differentiation, experiments

A
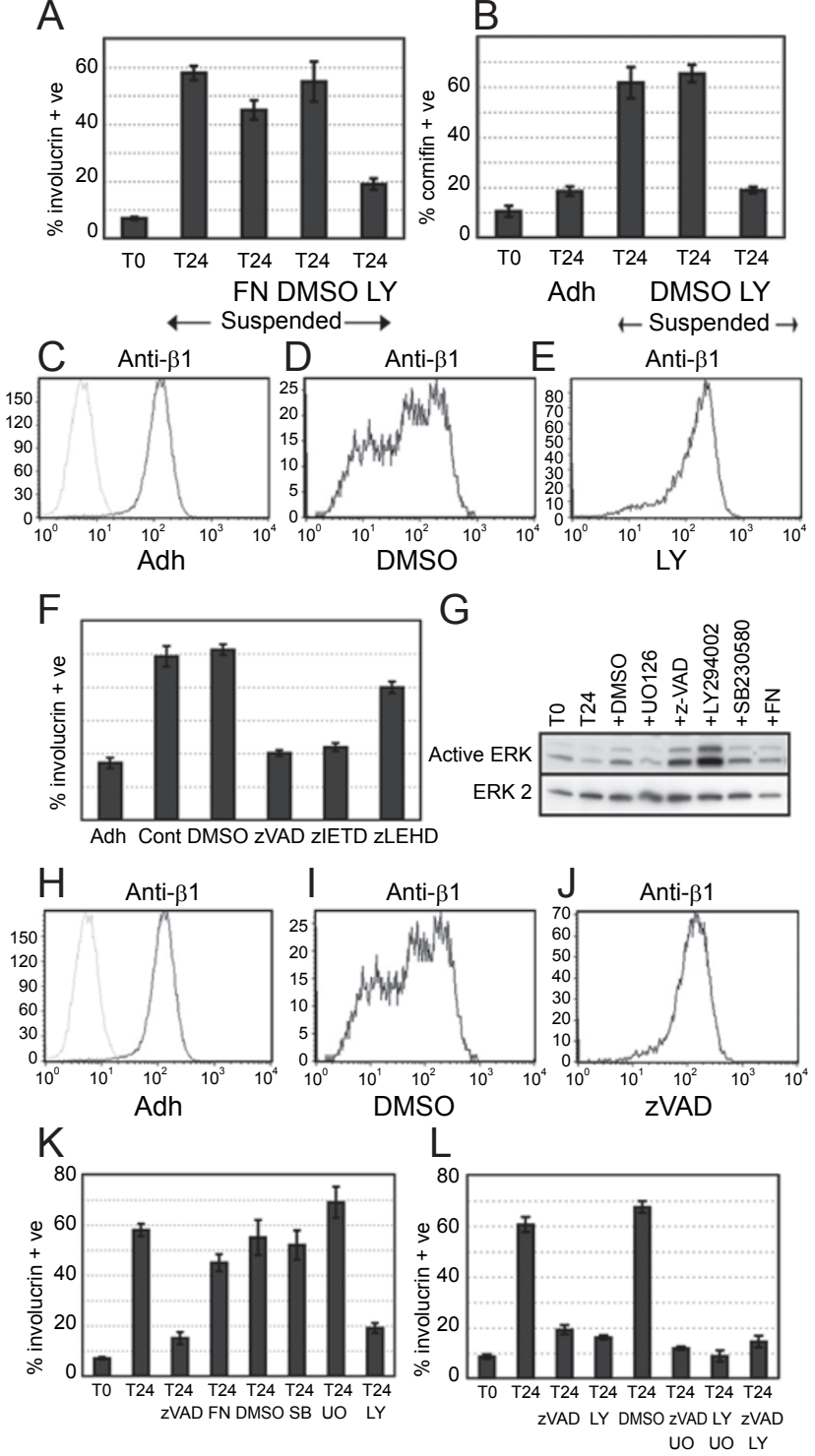

Figure 3 Inhibition of suspension-induced terminal differentiation with inhibitors of PI3-kinase or caspases. Cells that had been in suspension for $24 \mathrm{~h}$ (T24) were compared with adherent (Adh) cells (T0; Adh). \% involucrin (A, F, K, L) or cornifin (B) positive cells were determined. Cell surface $\beta 1$ integrin levels were examined (C-E, H-J) and the levels of total and active ERK MAPK (G). Left-hand peaks in (C, H) show fluorescence of cells labeled with secondary antibody alone, as a negative control. The effects of the following treatments were examined: 50 $\mu \mathrm{M}$ LY294002 (LY), DMSO (solvent control), $100 \mu \mathrm{g} / \mathrm{ml}$ fibronectin (FN), 50 (J) or 100 (F, K, L) $\mu \mathrm{M}$ zVAD-fmk (zVAD), $10 \mu \mathrm{M} \mathrm{SB}$ 203580 (SB), $10 \mu \mathrm{M}$ UO126 (UO), $100 \mu \mathrm{M}$ z-VAD-IETD (zIETD) or $100 \mu \mathrm{M}$ z-VAD-LEHD (zLEHD). Error bars show SEM. 
using either caspase 8 or 9 specific inhibitors were performed (Figure 3F). The caspase 8 inhibitor (z-IETDfmk) suppressed suspension-induced differentiation as efficiently as z-VAD-fmk, while the caspase 9 specific inhibitor (z-LEHD-fmk) partially suppressed suspensioninduced differentiation. Thus it appears that both pathways contribute to the differentiation process, with the caspase 8-dependent pathway being more important. However, additional experimental approaches will be necessary to clearly delineate the relative contributions of caspase 8 and 9 .

Other signalling pathways that have been implicated in the regulation of keratinocyte differentiation are the Ras-Raf-MEK-Erk cascade [12, 27, 28] and the ASK1-
MEKK1-p38 pathway [29]. Integrin-dependent Erk activation is associated with maintenance of the human epidermal stem cell compartment in culture [28]. Inhibitors of MEK1 (UO126, $10 \mu \mathrm{M}$ ) and p38 MAPK (SB 203580, $10 \mu \mathrm{M})$ did not inhibit suspension-induced involucrin expression (Figure $3 \mathrm{~K}$ ). We did, however, observe that when suspended keratinocytes were treated with z-VADfmk or LY294002 there was a higher level of ERK activation than in untreated cells or cells suspended in the presence of fibronectin (Figure 3G). The enhanced ERK activation reflected a failure of the cells to down-regulate ERK rather than an induction in suspension, as shown by comparing levels of active ERK in adherent and suspended cells (data not shown).
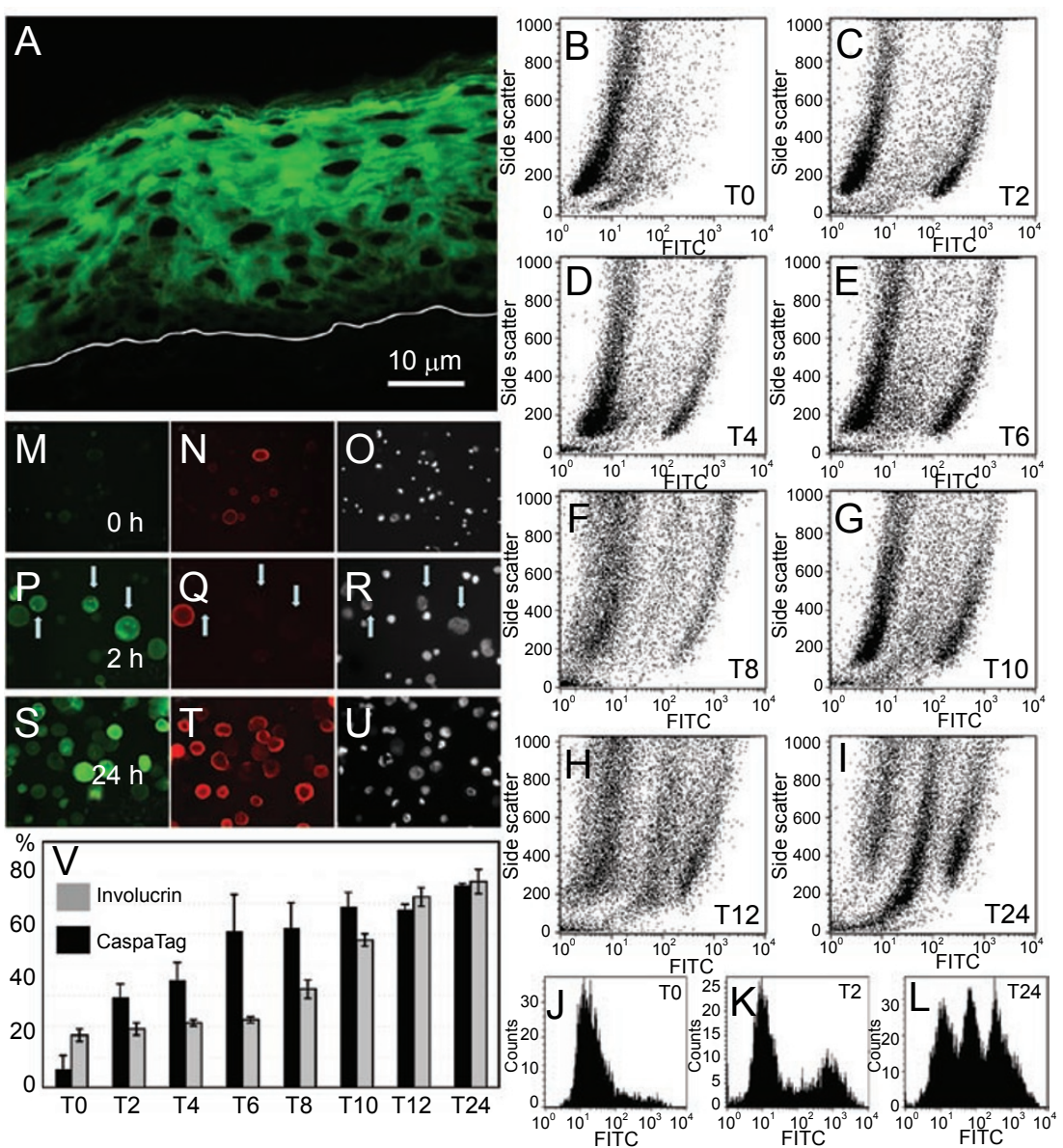

Figure 4 Kinetics of caspase activation during terminal differentiation. (A) CaspaTag ${ }^{\mathrm{TM}}$ labeling of human epidermis. Position of basement membrane is indicated. Scale bar: $10 \mu \mathrm{m}$. (B-L) Flow cytometry of CaspaTag ${ }^{\text {TM }}$-labeled keratinocytes that had been freshly harvested (TO) or held in suspension for the number of hours shown. Dead cells were gated out with propidium iodide staining. (M-U) Fluorescence microscopy of cells labeled with CaspaTag ${ }^{\mathrm{TM}}$ (green), involucrin (red) and Hoechst (white). Cells were freshly harvested (M-O) or held in suspension for $2 \mathrm{~h}(\mathbf{P}-\mathbf{R})$ or $24 \mathrm{~h}$ (S-U). Arrows show cells that are strongly labeled with CaspaTag ${ }^{\mathrm{TM}}$, are involucrin negative and have large, pale nuclei. Note that cells that are less intensely labeled with CaspaTag $^{\mathrm{TM}}$ are involucrin-positive cells with smaller, more intensely stained nuclei. (V) Total numbers of CaspaTag ${ }^{{ }^{\mathrm{TM}}}$-positive (strong and intermediate labeling) and involucrin-positive cells with time in suspension. The data represent quantitation of the type of flow cytometry data in (B-L) and are combined from three independent experiments \pm standard error of the mean. 
To determine whether the inhibition of differentiation by z-VAD-fmk and LY294002 was dependent on sustained ERK activation, we treated suspended cells with the UO126 inhibitor in combination with z-VAD-fmk or LY294002. As shown in Figure 3L, UO126 did not overcome z-VAD-fmk or LY294002 inhibition of terminal differentiation, even though it prevented sustained ERK activation (data not shown). These experiments show that sustained ERK activation is not required for the block in differentiation caused by caspase or PI3-kinase inhibition.

Changes in caspase activity occur within 2 h of placing keratinocytes in suspension

To examine in vivo caspase activity, sections of normal human epidermis were labeled with CaspaTag ${ }^{\mathrm{TM}}$, a substrate that fluorescently labels activated caspases. CaspaTag $^{\mathrm{TM}}$ did not label cells in the basal layer, but labeled all the suprabasal layers (Figure 4A). This prompted us to use CaspaTag ${ }^{\mathrm{TM}}$ to examine the kinetics of caspase activation during suspension-induced differentiation. The majority of cells freshly harvested from adherent cultures (T0) were unlabeled (Figure 4B, 4J, 4M-4O and 4V), but by $2 \mathrm{~h} 40 \%$ of cells were positive (Figure 4C, 4P$4 \mathrm{R}, 4 \mathrm{~K}$ and $4 \mathrm{~V}$ ). The proportion of CaspaTag ${ }^{\mathrm{TM}}$-labeled cells continued to increase when monitored for up to 24 $\mathrm{h}$ in suspension (Figure 4D-4I, 4L, 4S-4U and 4V). The increase in CaspaTag ${ }^{\mathrm{TM}}$ labeling preceded the increase in involucrin-positive cells, which did not start until $8 \mathrm{~h}$ of suspension (Figure 4V). During keratinocyte differentiation in suspension the nucleus is known to enlarge and become paler, before disappearing completely [5]; nuclear swelling is also a feature of necrotic cell death [30]. The nuclear changes were already apparent within 2 $\mathrm{h}$ of placing keratinocytes in suspension (Figure 4O, 4R and $4 \mathrm{U})$.

Flow cytometry identified two subpopulations of cells with distinct levels of CaspaTag ${ }^{\mathrm{TM}}$ labeling. At time zero there was a single major peak of unlabeled cells (Figure $4 \mathrm{~B}$ and $4 \mathrm{~J}$ ) and at $2 \mathrm{~h}$ a peak of intensely labeled cells was detected (Figure 4C and 4K). By $24 \mathrm{~h}$, the second peak of CaspaTag ${ }^{\mathrm{TM}}$-labeled cells, which has a fluorescence intensity intermediate between the unlabeled and strongly labeled populations, was as abundant as the first peak (Figure 4I and 4L).

The intermediate peak of CaspaTag ${ }^{\mathrm{TM}}$ labeling was detectable from time zero onwards, but did not increase in abundance until after $10 \mathrm{~h}$ in suspension (Figure 4B4I). A population with intermediate CaspaTag ${ }^{\mathrm{TM}}$ labeling was also detectable by fluorescence microscopy (Figure $4 \mathrm{~S}-4 \mathrm{U})$. These cells tended to be involucrin positive and to have normal nuclear morphology (Figure 4S-4U). In
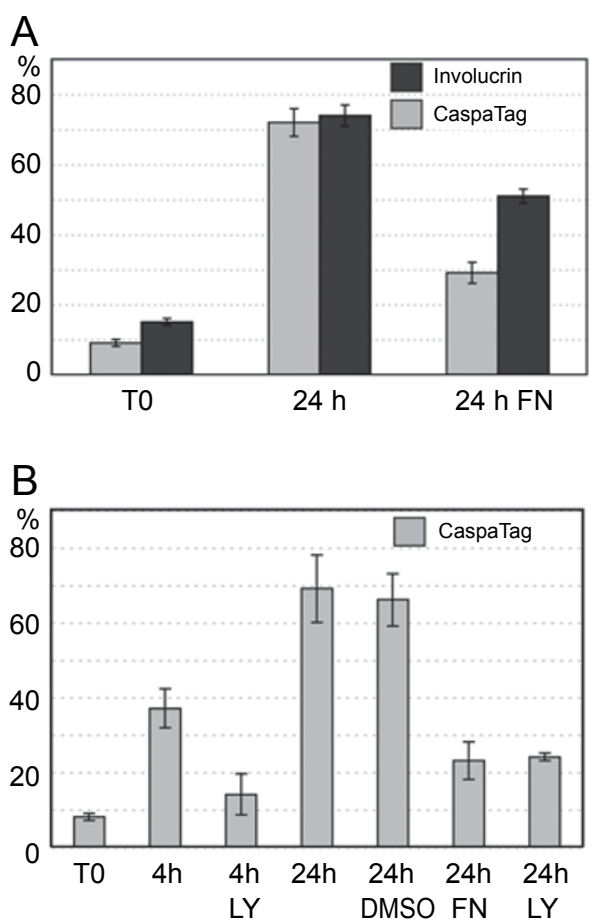

Figure 5 Effects of PI3-kinase inhibition and fibronectin on caspase activation and involucrin expression. Adherent (T0) keratinocytes were compared with cells that had been incubated in suspension for $4 \mathrm{~h}$ or $24 \mathrm{~h}$ in the presence or absence of $100 \mu \mathrm{g} /$ ml fibronectin (FN), $100 \mu \mathrm{M}$ LY294002 (LY) or DMSO (diluent control). $\%$ Involucrin-positive and Caspatag ${ }^{{ }^{\mathrm{TM}}}{ }_{\text {-labeled cells are }}$ shown \pm SEM.

contrast, the cells that were most brightly labeled with CaspaTag $^{\mathrm{TM}}$ tended to have nuclear changes but no involucrin staining. This suggests that the initial stages of terminal differentiation are associated with high caspase activity and nuclear enlargement, while at later stages caspase activity is somewhat reduced. Alternatively, caspases that bind CaspaTag ${ }^{\mathrm{TM}}$ with different affinities may be activated early and late in terminal differentiation.

To investigate whether suspension-induced caspase activation was subject to the same regulation as induction of involucrin expression, we examined the effects of incubation with fibronectin or LY294002 (Figure 5). Both CaspaTag $^{\text {TM }}$ labeling and involucrin expression were reduced by the addition of fibronectin or LY294002. We noted that CaspaTag ${ }^{\mathrm{TM}}$ labeling was more sensitive than involucrin induction to inhibition by fibronectin (Figure $5 \mathrm{~A}$ ), and that the increase in CaspaTag ${ }^{\mathrm{TM}}$ labeling that occurred by $4 \mathrm{~h}$ in suspension was inhibited by LY294002. This would be consistent with previous reports that caspases are activated by unbound integrins [8]. 

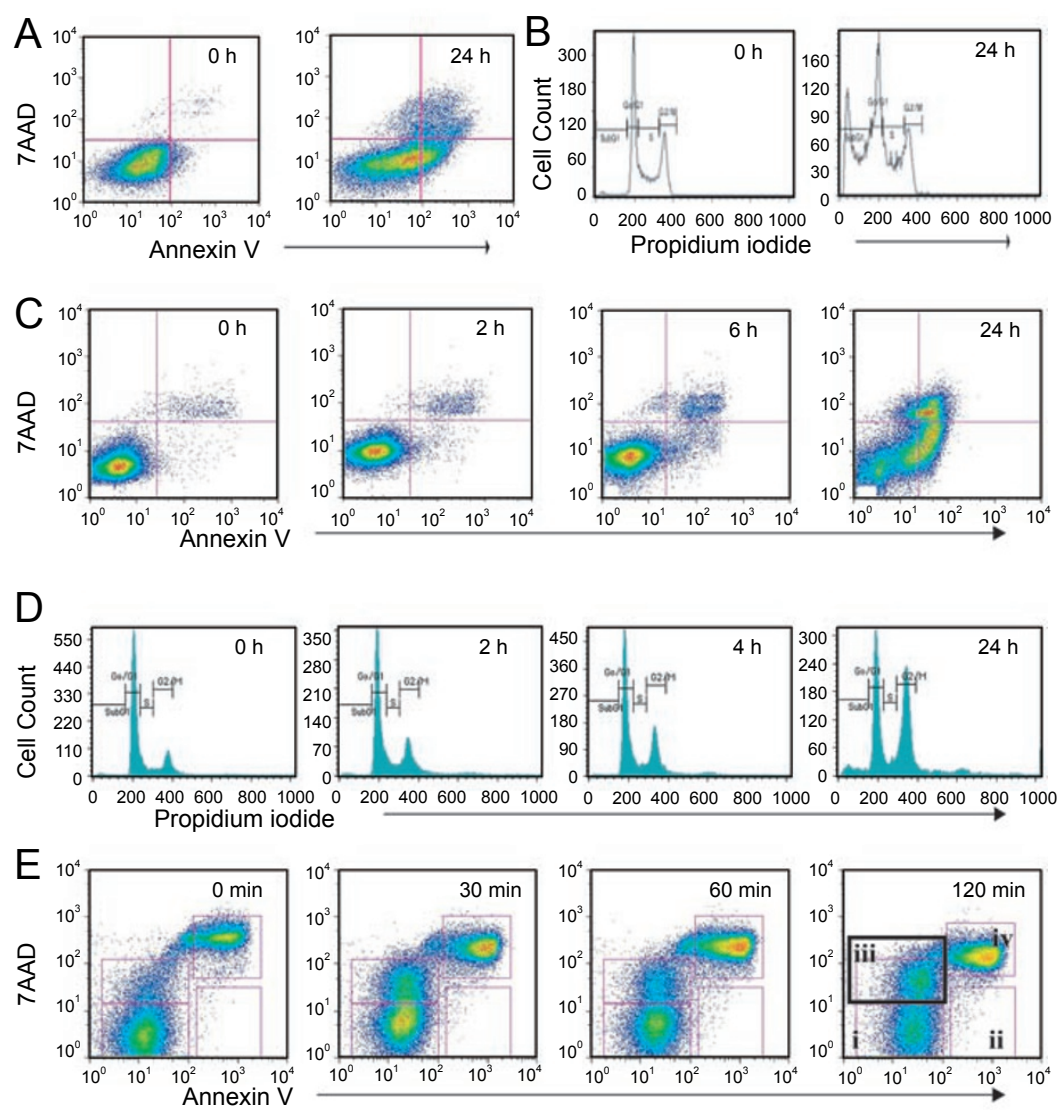

Figure 6 Keratinocytes undergoing suspension-induced terminal differentiation do not undergo DNA fragmentation or expose phosphatidylserine. (A, C, E) Flow cytometry of cells labeled with 7-AAD and Annexin V. (B, D) Flow cytometry profiles of DNA content. Gates show (left to right) sub-G1, G1, S, G2+M DNA content. (A) MDCK cells induced to undergo apoptosis in suspension; $(\mathbf{B}, \mathbf{C})$ adherent keratinocytes induced to undergo apoptosis by treatment with $1 \mu \mathrm{M}$ staurosporine; (D, E) keratinocytes undergoing terminal differentiation in suspension. In (E) box i denotes cells that are 7-AAD and Annexin $V$ negative; box ii denotes Annexin V-positive 7-AAD-negative cells; box iii denotes 7-AAD-positive, Annexin V-negative cells; and box iv denotes double-positive cells.

Suspension-induced differentiation is distinct from apoptosis, with no phosphatidylserine membrane flipping

Early in apoptosis the phospholipid asymmetry of the plasma membrane is lost, exposing phosphatidylserine on the outer layer of the membrane. The early activity of caspases in suspension-induced differentiation led us to examine whether differentiating keratinocytes undergo phosphatidylserine flipping, as measured by multiparametric flow cytometry for Annexin V-positive cells in combination with other markers including the nuclear dye 7-aminoactinomycin D (7-AAD) (Figure 6). Although the emission wavelengths of 7-AAD and Annexin $\mathrm{V}^{\mathrm{Alexa647}}$ are the same $(660 / 20 \mathrm{~nm})$, they are excited by different wavelengths and so can be examined together. Little compensation (correction of fluorescence signals) is needed between 7-AAD and AnnexinV $\mathrm{V}^{\text {Alexa647 }}$, because $7-\mathrm{AAD}$ is excited by the $488 \mathrm{~nm}$ blue argon laser, which does not excite Alexa 647; and Alexa 647 is excited by the $633 \mathrm{~nm}$ red diode laser, which does not excite 7-AAD.

We first examined Annexin V staining in cells undergoing apoptosis. MDCK cells provide a positive control for suspension-induced apoptosis [18], whereas staurosporine is known to induce apoptosis of adherent primary human keratinocytes at certain concentrations [31]. Freshly detached MDCK cells were largely negative for Annexin $\mathrm{V}$ and for the nuclear dye 7-AAD, but by $24 \mathrm{~h}$ in suspension a double-positive population was detected; this provides a positive control for apoptosis of suspended cells (Figure 6A). Double-positive cells were also detected when adherent keratinocytes were treated with $1 \mu \mathrm{M}$ staurosporine for $24 \mathrm{~h}$ (Figure 6C). We confirmed that staurosporine induced keratinocyte apoptosis by determining that cells with a sub-G1 DNA content were present (Figure 6B).

In contrast to keratinocytes undergoing staurosporine- 
induced apoptosis, keratinocytes undergoing suspensioninduced terminal differentiation accumulated in G2/M of the cell cycle, with some evidence of polyploidy; few cells entered the sub-G1 fraction (Figure 6D; [32]). In the early phases of suspension-induced differentiation, cells that were permeable to the nuclear dye 7-AAD (and Hoechst; data not shown) and positive for Annexin $\mathrm{V}$ were readily detected (box iv) (Figure 6E). However, there was also a distinct population of cells that were $7-\mathrm{AAD}$ positive and Annexin $\mathrm{V}$ negative (box iii) (Figure $6 \mathrm{E}$ ). Such cells were not detected in apoptotic cells (Figure $6 \mathrm{C})$.

Suspension-induced differentiation involves early cytochrome c release and loss of mitochondrial membrane potential
It has been suggested previously that keratinocyte differentiation uses the mitochondrial-dependent cell death machinery [33]. To examine whether the CaspaTag ${ }^{\text {TM }}$ activity seen within $2 \mathrm{~h}$ of cell suspension was indicative of apoptosis, we performed a multiparametric apoptosis assay on unfixed, nonpermeabilized keratinocytes. Three markers (cytochrome c release, loss of mitochondrial membrane potential and increase in reactive oxygen species) were evaluated by flow cytometry. Direct determination of cytochrome $c$ release cannot be carried out on living cells; instead we used nAO fluorescence as an indirect measure. $\mathrm{nAO}$ measures cardiolipin peroxidation; cardiolipin anchors cytochrome $c$ in mitochondria, and thus a decrease in nAO fluorescence can be attributed to cytochrome c release [34]. Cardiolipin peroxidation can also be caused directly by ROS production [35].
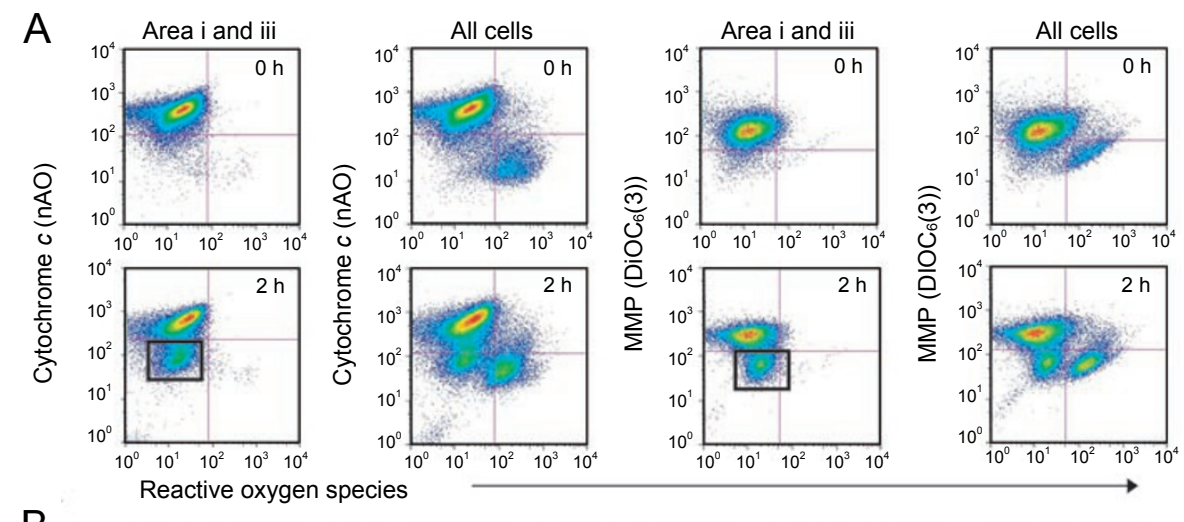

B
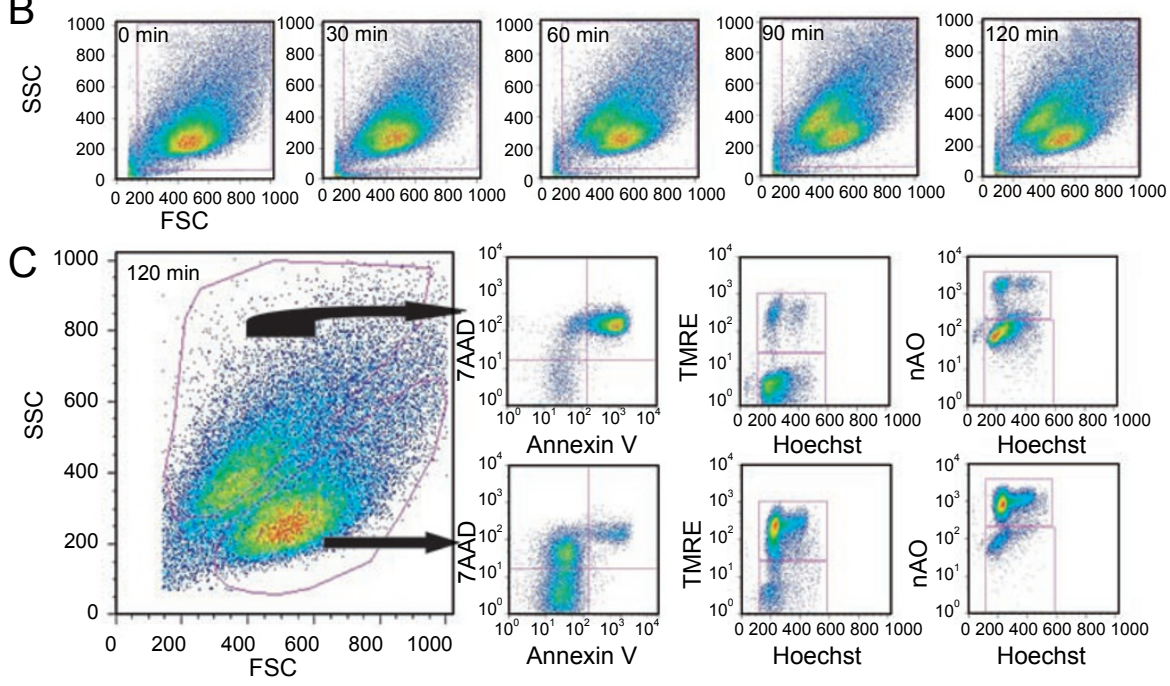

Figure 7 Cytochrome $c$ release and loss of mitochondrial membrane potential during suspension-induced terminal differentiation. (A) Keratinocytes were freshly harvested $(0 \mathrm{~h})$ or held in suspension for $2 \mathrm{~h}$. The total population (all cells) is compared with cells corresponding to areas i (7-AAD, Annexin $V$ double negative) and iii (7-AAD positive, Annexin $V$ negative) of Figure 6E. (B) Increase in side scatter (SSC) of keratinocytes held in suspension for up to $120 \mathrm{~min}$. (C) The cells with higher SSC at $120 \mathrm{~min}$ are 7-AAD positive, and exhibit loss of cytochrome $c$ and mitochondrial membrane potential (top row), while the cells with lower SCC (bottom row) tend to have lower 7-AAD staining with no loss of cytochrome $c$ or mitochondrial membrane potential. 
Freshly isolated, unfractionated cell populations contained a small subpopulation of cells that were positive for all three markers (Figure 7A). However, by $2 \mathrm{~h}$ in suspension a more abundant subpopulation appeared that showed reduced nAO fluorescence and loss of mitochondrial membrane potential but did not show an increase in reactive oxygen species (boxed in Figure 7A). Since ROS production was not increased we deduce that the change in nAO indicates cytochrome $c$ release. These cells corresponded to the cells that were 7-AAD positive and Annexin $\mathrm{V}$ negative (box iii in Figure 6E) (Figure $7 \mathrm{~A})$.

As keratinocytes undergo terminal differentiation they increase in forward and side scatter [22]. At $2 \mathrm{~h}$ in suspension we observed an intermediate cell population that had increased side scatter without increased forward scatter (Figure 7B). These cells corresponded to the cells that had become permeable to 7-AAD with loss of mitochondrial membrane potential and release of cytochrome $c$ (Figure 7C). Thus, we can distinguish cells that are committed to terminal differentiation as being 7-AAD positive, Annexin $\mathrm{V}$ negative, with loss of mitochondrial membrane potential, release of cytochrome $c$, no increase in reactive oxygen species and an increase in side but not forward scatter.

\section{Discussion}

Our experiments set out to delineate events in commitment of human epidermal keratinocytes to terminal differentiation. We examined the role of Akt, the temporal activation of apoptotic machinery and the extent to which commitment is a modified form of apoptosis.

In agreement with several previous reports [12-14], we found that Akt was activated on initiation of terminal differentiation and that PI3K activity was required for differentiation to proceed. Akt activation did not cause immediate withdrawal from the cell cycle, because cells were still able to found colonies, but rather triggered a transition from the stem into the transit amplifying compartment. Inhibition of PI3-kinase prevented not only induction of spinous layer differentiation markers in suspension, but also the down-regulation of integrin expression that occurs on departure from the basal layer. There was no evidence for a role of the other signaling pathways tested, in particular ERK MAPK signaling, although integrin-dependent activation of ERK MAPK is involved in maintenance of the stem cell compartment [28].

Previous work has demonstrated that caspases are involved in the later stages in epidermal differentiation [33, $36,37]$. We found, however, that caspases were activated as soon as cells left the basal layer of the epidermis and within $2 \mathrm{~h}$ of placing them in suspension. Inhibition of caspases blocked suspension-induced terminal differentiation, as measured both by induction of involucrin and by down-regulation of surface $\beta 1$ integrins. Caspases 8 and 9 were involved. Caspase activation could be blocked by integrin ligation with fibronectin or by inhibition of PI3kinase, suggesting Akt may act upstream of caspase activation. In suspended squamous cell carcinoma cells, caspase activation results in decreased Akt phosphorylation [18], raising the possibility of a feedback loop involving caspase and Akt activation.

The different levels of CaspaTag ${ }^{\mathrm{TM}}$ staining we observed at 2 and $24 \mathrm{~h}$ of suspension may indicate activation of different caspases or different levels of activation, perhaps by a negative feedback mechanism as differentiation progresses. Many caspases are expressed in keratinocytes, including caspase 1, 2, 3, 4, 6, 7, 8, 9, 11 and 14 [37-39]. Caspases 3 and 14 have been implicated in the later stages of terminal differentiation [37, 40]. A possible model based on the concept of negative feedback is that there is early activation of caspase 8 by unbound integrins [8]; caspase 8 activation could then be amplified by a caspase 9-dependent process as seen in suspension-induced apoptosis [41]. Akt activation, which increases from $6 \mathrm{~h}$ after suspension $[17,18]$, could control the caspase activity and prevent the induction of apoptosis [41]. This would reduce caspase activity in cells undergoing the later stages of differentiation, allowing ordered assembly of the cornified layers. Consistent with our finding that caspase activation plays a role in keratinocyte differentiation, there are recent reports that caspase 3 mediates differentiation of embryonic stem cells and hematopoietic stem cells $[42,43]$.

We conclude that Akt and caspase activity have dual roles in keratinocyte differentiation, stimulating an initial commitment to differentiate and subsequently allowing keratinocytes to progress through the later stages of differentiation. The early caspase activity is associated with other apoptotic mechanisms including cytochrome c release and loss of mitochondrial membrane potential. However, the processes can be distinguished by the lack of phosphatidylserine flipping and generation of reactive oxygen species, which may indicate that commitment is associated with changes in membrane permeability rather than a classic apoptotic process.

We previously presented evidence for a model in which Akt activation in keratinocytes provides the survival signal necessary for initiation of terminal differentiation in suspension $[17,18]$. We further showed that tumour cells that failed to differentiate underwent programmed cell death through a failure to activate Akt, but 
that this could be over-ridden by upregulated expression of the $\alpha v \beta 6$ integrin [18]. Our finding that commitment to differentiation is associated with an increase in side scatter will allow us to isolate committed cells in order to investigate how the PI3-kinase-Akt pathway interacts with other signalling pathways that control exit from the epidermal stem cell compartment.

\section{Materials and Methods}

\section{Cell culture}

Primary human keratinocytes [strain km, passages $4(\mathrm{~km} 4), 5$ and 6] were isolated from neonatal foreskin and cultured in the presence of a feeder layer of J2-3T3 cells in FAD medium containing $10 \% \mathrm{FCS}, 0.5 \mu \mathrm{g} / \mathrm{ml}$ hydrocortisone, $5 \mu \mathrm{g} / \mathrm{ml}$ insulin, $10^{-10}$ $\mathrm{M}$ cholera toxin and $10 \mathrm{ng} / \mathrm{ml} \mathrm{EGF}$, as described previously [44]. MDCK cells were cultured in DMEM containing 10\% FCS.

\section{Retroviral infection}

The A2AktER and myrAktER constructs [24] cloned into the retroviral vector pWZLneo were kind gifts of Julian Downward (CR-UK London Research Institute). Retroviruses were packaged using a two-step procedure involving transient transfection of ecotropic Phoenix-AM12 cells and infection of amphotropic AM12 cells with the viral supernatant [45]. Keratinocyte cultures were transduced with the supernatant from the amphotropic AM12 producer cells [44]. After $24 \mathrm{~h}$ the cells were selected with neomycin $(2.0 \mathrm{mg} / \mathrm{ml})(\mathrm{G} 418$, Geneticin; Gibco). Activation of A2AktER and myrAktER was achieved by adding $100 \mathrm{nM} 4-\mathrm{OHT}$ to the culture medium.

\section{Clonogenicity assays}

In all, 500 keratinocytes were plated per $60 \mathrm{~mm}$ dish. After 14 days the cultures were fixed in formaldehyde for $10 \mathrm{~min}$ and stained with 1\% Rhodanile Blue. All colonies (that is, consisting of 2 cells or more) were scored. Colony-forming efficiency was calculated as the percentage of plated cells that formed colonies. Abortive colonies were defined as colonies that contained fewer than 40 cells [22,28]. All experiments were performed in triplicate and more than 100 colonies were scored per experimental condition.

\section{Antibodies}

Antibodies against the following proteins were used: Akt (9272; Cell Signalling Technology), phospho-Akt ${ }^{473}$ (44-622; Cell Signalling Technology and Biosource), Erk2 (sc-1647; Santa Cruz Biotechnology), $\beta 1$ integrins (P5D2), involucrin (SY5) and cornifin (SQ37C). Species-specific secondary antibodies conjugated to Alexa 488 or Alexa 594 were purchased from Molecular Probes, and HRP-conjugated second antibodies were purchased from Amersham.

\section{Immunofluorescence labeling of epidermis}

Frozen sections of neonatal human foreskin were fixed in $4 \%$ formaldehyde for $10 \mathrm{~min}$. Sections were incubated for $60 \mathrm{~min}$ at room temperature with antibodies diluted in blocking buffer (PBS, $0.1 \%$ Triton X-100, $2 \%$ BSA, $4 \%$ goat serum). Nuclear counterstaining was performed using propidium iodide or Hoechst 33342.
Epidermal wholemounts were prepared and labeled as described previously [21]. Sections and wholemounts were analyzed on a Zeiss 510 confocal microscope.

\section{Suspension-induced terminal differentiation}

Cells were suspended in medium containing $3.5 \mathrm{~g} / 200 \mathrm{ml}$ methyl cellulose (Sigma), as described previously [9]. The following inhibitors were used at the concentrations indicated: z-VAD-fmk (100 $\mu \mathrm{M}$; ICN Pharmaceuticals Inc.), z-LIEH-fmk (100 $\mu \mathrm{M}$; Sigma), z-LEHD-fmk (100 $\mu$ M; Sigma), LY294002 (50 $\mu$ M; Sigma), SB203580 (10 $\mu \mathrm{M}$; Sigma), U0126 (10 $\mu \mathrm{M}$; Promega) or $100 \mu \mathrm{g} /$ $\mathrm{ml}$ fibronectin (Sigma). To measure the proportion of keratinocytes expressing involucrin or cornifin, cells were recovered by dilution of the methyl cellulose supplemented medium, air dried onto coverslips, fixed in $4 \%$ formaldehyde for $10 \mathrm{~min}$ at room temperature, permeabilized in methanol on ice for $10 \mathrm{~min}$ and then labeled with the appropriate antibodies [46]. At least 300 cells per experimental condition were scored for involucrin or cornifin expression.

\section{Western blotting}

Cells were lysed on ice in a modified RIPA buffer containing $50 \mathrm{mM}$ Tris, $\mathrm{pH} 8.0,150 \mathrm{mM} \mathrm{NaCl}, 1 \% \mathrm{NP}-40,0.1 \%$ SDS, $0.5 \%$ deoxycholate, $5 \mathrm{mM}$ EDTA and $1 \%$ Triton X-100, with protease and phosphatase inhibitor cocktails (Sigma) [47]. Soluble proteins were resolved by SDS-PAGE on 10\% Laemmli gels and transferred onto Millipore Immobilon PVDF membranes. Primary antibody probed blots were visualized with appropriate horseradish peroxidase-coupled secondary antibodies using enhanced chemiluminescence (ECL; Amersham) for detection [47].

\section{Detection of active caspases}

Cells were labeled with the Intergen CaspaTag ${ }^{\text {TM }}$ Pan Fluorescein Caspase Activity Kit, which uses a carboxyfluoresceinlabeled caspase inhibitor to bind irreversibly to active caspases. Single-cell suspensions and frozen skin sections were incubated according to the supplier's instructions with CaspaTag ${ }^{\mathrm{TM}}$ at $37^{\circ} \mathrm{C}$ for $1 \mathrm{~h}$. Cell suspensions were analyzed by flow cytometry or immunofluorescence microscopy.

\section{Flow cytometry}

Single-cell suspensions of keratinocytes $\left(10^{6} / \mathrm{ml}\right)$ were labeled with anti- $\beta 1$ integrin and fluorescent secondary antibody on ice and then fixed with $4 \%$ paraformaldehyde prior to analysis. When DNA analysis was carried out, live cells were incubated with $10 \mu \mathrm{g} / \mathrm{ml}$ Hoechst 33342 (Sigma) for $45 \mathrm{~min}$ at $37{ }^{\circ} \mathrm{C}$ before staining for apoptotic markers.

To detect apoptotic markers, live cells were incubated with $50 \mathrm{nM}$ nonyl acridine orange (nAO; Molecular Probes) or $4 \mathrm{nM}$ $\mathrm{DiOC}_{6}(3)$ (Molecular Probes) and $5 \mu \mathrm{M}$ dihyrdoethidium (HE, Molecular Probes) for $30 \mathrm{~min}$ at $37^{\circ} \mathrm{C}$ [48]. A separate aliquot of cells was also incubated with $40 \mathrm{nM}$ tetramethylrhodamine ethyl ester (TMRE; Molecular Probes) for $20 \mathrm{~min}$ at $37^{\circ} \mathrm{C}$. Cells were then washed and incubated with Annexin $\mathrm{V}^{\text {Alexa647 }}$ (Molecular Probes) and $200 \mathrm{nM}$ DAPI (Sigma Chemicals) or $4 \mu \mathrm{g} / \mathrm{ml}$ 7-AAD (Sigma Chemicals) in calcium-rich buffer (Becton Dickinson) for $15 \mathrm{~min}$ at room temperature. TMRE was excited at $488 \mathrm{~nm}$ and detected at $580 / 40 \mathrm{~nm}$.

Cell samples (50 000 events) were analyzed on a Becton Dickinson LSR II fitted with 405, 488, 350-360 and $633 \mathrm{~nm}$ lasers. 
$\mathrm{nAO}$ and $\mathrm{DiOC}_{6}(3)$ emissions were detected using a 530/25 nm filter; HE emission was detected using a $610 / 20 \mathrm{~nm}$ filter; DAPI emission was detected with a 440/40 nm filter; and Annexin $\mathrm{V}^{\mathrm{Al}-}$ exa647 emission was detected with a $660 / 20 \mathrm{~nm}$ filter. The DNA content of ethanol-fixed cells stained with $50 \mu \mathrm{g} / \mathrm{ml}$ propidium iodide in the presence of RNase A $(100 \mu \mathrm{g} / \mathrm{ml})$ was determined with a FACSCalibur flow cytometer.

\section{Acknowledgments}

This work was supported by Cancer Research UK. SMJ was the recipient of a Medical Research Council Clinical Fellowship. DHC was the recipient of a European Union Marie Curie Fellowship. FMW gratefully acknowledges the support of the University of Cambridge and Hutchison Whampoa Ltd.

\section{References}

1 Jones PH, Simons BD, Watt FM. Sic Transit Gloria. Farewell to the epidermal transit amplifying cell? Cell Stem Cell 2007; 1:371-381.

2 Watt F. Epidermal stem cells. In: Marshak DR, Gottlieb D, eds. Stem cell biology. Cold Spring Harbor: Cold Spring Harbor Laboratory Press, 2001: 439-453.

3 Green H. Terminal differentiation of cultured human epidermal cells. Cell 1977; 11:405-416.

4 Adams J, Watt FM. Fibronectin inhibits the terminal differentiation of human keratinocytes. Nature 1989; 340:307-309.

5 Gandarillas A, Goldsmith LA, Gschmeissner S, Leigh IM, Watt FM. Evidence that apoptosis and terminal differentiation of epidermal keratinocytes are distinct processes. Exp Dermatol 1999; 8:71-79.

6 Watt FM, Kubler MD, Hotchin NA, Nicholson LJ, Adams JC. Regulation of keratinocyte terminal differentiation by integrinextracellular matrix interactions. J Cell Sci 1993; 106:175-182.

7 Polakowska R, Piacentini M, Bartlet R, Goldsmith LA, Haake AR. Apoptosis in human skin development: morphogenesis, periderm, and stem cells. Dev Dyn 1994; 199:1441-1447.

8 Stupack D, Puente XS, Boutsaboualoy S, Storgard CM, Cheresh DA. Apoptosis of adherent cells by recruitment of caspase- 8 to unligated integrins. J Cell Biol 2001; 155:459570 .

9 Levy L, Broad S, Diekmann D, Evans RD, Watt FM. $\beta 1$ integrins regulate keratinocyte adhesion and differentiation by distinct mechanisms. Mol Biol Cell 2000; 11:453-466.

10 Watt FM. Role of integrins in regulating epidermal adhesion, growth and differentiation. EMBO J 2002; 21:3919-3926.

11 Sayama K, Yamasaki K, Hanakawa Y, et al. Phosphatidylinositol 3-kinase is a key regulator of early phase differentiation in keratinocytes. $J$ Biol Chem 2002; 277:40390-40396.

12 Dajee M, Tarutani M, Deng H, Cai T, Khavari PA. Epidermal Ras blockade demonstrates spatially localized Ras promotion of proliferation and inhibition of differentiation. Oncogene 2002; 21:1527-1538.

13 Calautti E, Li J, Saoncella S, Brissete JL, Goetinck PF. Phosphoinositide 3-kinase signalling to Akt promotes keratinocyte differentiation versus death. J Biol Chem 2005; 280:3285632865 .

14 Thrash B, Menges CW, Pierce RH, McCance DJ. AKT1 pro- vides an essential survival signal for differentiation and stratification of primary human keratinocytes. J Biol Chem 2006; 281:12155-12162.

15 Peng XD, Xu PZ, Chen ML, et al. Dwarfism, impaired skin development, skeletal muscle atrophy, delayed bone development, and impeded adipogenesis in mice lacking Akt1 and Akt2. Genes Dev 2003; 17:1352-1365.

16 Murayama K, Kimura T, Tarutani M, et al. Akt activation induces epidermal hyperplasia and proliferation of epidermal progenitors. Oncogene 2007; 26:4882-4888.

17 Janes SM, Ofstad TA, Campbell DH, Watt FM, Prowse DM. Transient activation of FOXN1 in keratinocytes induces a transcriptional programme that promotes terminal differentiation: contrasting roles of FOXN1 and Akt. J Cell Sci 2004; 117:4157-4168

18 Janes SM, Watt FM. Switch from $\alpha v \beta 5$ to $\alpha v \beta 6$ integrin expression protects squamous cell carcinomas from anoikis. $J$ Cell Biol 2004; 166:419-431.

19 Umeda J, Sano S, Kogawa K, et al. In vivo cooperation betwen Bcl-xL and the phosphoinositide 3-kinase-Akt signaling pathway for the protection of epidermal keratinocytes from apoptosis. FASEB J 2003; 17:610-620.

20 Watt FM, Green H. Stratification and terminal differentiation of cultured epidermal cells. Nature 1982; 295:434-436.

21 Jensen UB, Lowell S, Watt FM. The spatial relationship between stem cells and their progeny in the basal layer of human epidermis: a new view based on whole-mount labelling and lineage analysis. Development 1999; 126:2409-2418.

22 Jones PH, Watt FM. Separation of human epidermal stem cells from transit amplifying cells on the basis of differences in integrin function and expression. Cell 1993; 73:713-724.

23 Strachan LR, Scalapino KJ, Lawrence HJ, Ghadially R. Rapid adhesion to collagen isolates murine keratinocytes with limited long-term repopulating ability in vivo despite high clonogenicity in vitro. Stem Cells 2008; 26:235-243.

24 Kohn AD, Barthel A, Kovacina KS, et al. Construction and characterization of a conditionally active version of the serine/ threonine kinase Akt. J Biol Chem 1998; 273:11937-11943.

25 Frisch S. Evidence for a function of death-receptor-related, death-domain-containing proteins in anoikis. Curr Biol 1999; 9:1047-1049.

26 Rytomaa M, Martins ML, Downward J. Involvement of FADD and caspase- 8 signalling in detachment-induced apoptosis. Curr Biol 1999; 9:1043-1046.

27 Cai T, Nishida K, Hirano T, Khavari PA. Gab1 and SHP-2 promote Ras/MAPK regulation of epidermal growth and differentiation. J Cell Biol 2002; 159:103-112.

28 Zhu AJ, Haase I, Watt FM. Signaling via $\beta 1$ integrins and mitogen-activated protein kinase determines human epidermal stem cell fate in vitro. Proc Natl Acad Sci USA 1999; 96:67286733.

29 Sayama K, Hanakawa Y, Shirakata Y, et al. Apoptosis signalregulating kinase 1 (ASK1) is an intracellular inducer of keratinocyte differentiation. J Biol Chem 2001; 276:999-1004

30 Doonan F, Cotter TG. Morphological assessment of apoptosis Methods 2008; 44:200-204.

31 Honma M, Stubbs M, Collins I, Workman P, Aherne W, Watt FM. Identification of novel keratinocyte differentiation modulating compounds by high-throughput screening. J Biomol 
Screen 2006; 11:977-984.

32 Gandarillas A, Davies D, Blanchard JM. Normal and c-Mycpromoted human keratinocyte differentiation both occur via a novel cell cycle involving cellular growth and endoreplication. Oncogene 2000; 19:3278-3289.

33 Allombert-Blaise C, Tamiji S, Mortier L, et al. Terminal differentiation of human epidermal keratinocytes involves mitochondria- and caspase-dependent cell death pathway. Cell Death Differ 2003; 10:850-852.

34 Mileykovskaya E, Dowham W, Birke RL, Zheng D, Lutterodt L, Haines TH. Cardiolipin binds nonyl acridine orange by aggregating the dye at exposed hydrophobic domains on bilayer surfaces. FEBS Lett 2001; 507:187-190.

35 Nomura K, Imai H, Koumura T, Kobayashi T, Nakagawa Y. Mitochondrial phospholipid hydroperoxide glutathione peroxidase inhibits the release of cytochrome $\mathrm{c}$ from mitochondria by suppressing the peroxidation of cardiolipin in hypoglycaemiainduced apoptosis. Biochem J 2000; 351:183-193.

36 Weil M, Raff MC, Braga VM. Caspase activation in the terminal differentiation of human epidermal keratinocytes. Curr Biol 1999; 9:361-364.

37 Denecker G, Ovaere P, Vandenabeele P, Declercq W. Caspase-14 reveals its secrets. J Cell Biol 2008; 180:451-458.

38 Lippens S, Denecker G, Ovaere P, Vandenabeele P, Declercq W. Death penalty for keratinocytes: apoptosis versus cornification. Cell Death Differ 2005; 12:1497-1508.

39 Cohen G. Caspases: the executioners of apoptosis. Biochem J 1997; 326:1-16.

40 Eckhart L, Declercq W, Ban J, et al. Terminal differentiation of human keratinocytes and stratum corneum formation is associated with caspase-14 activation. J Invest Dermatol 2000; 115:1148-1151.

41 Rytomaa M, Lehmann K, Downward J. Matrix detachment in- duces caspase-dependent cytochrome c release from mitochodria: inhibition by $\mathrm{PKB} / \mathrm{Akt}$ but not Raf signalling. Oncogene 2000; 19:4461-4468.

42 Fujita J, Crane AM, Souza MK, et al. Caspase activity mediates the differentiation of embryonic stem cells. Cell Stem Cell 2008; 2:595-601.

43 Janzen V, Fleming HE, Riedt T, et al. Hematopoietic stem cell responsiveness to exogenous signals is limited by caspase- 3 . Cell Stem Cell 2008; 2:584-594.

44 Lowell S, Jones P, Le Roux I, Dunne J, Watt FM. Stimulation of human epidermal differentiation by Delta-Notch signalling at the boundaries of stem-cell clusters. Curr Biol 2000; 10:491500 .

45 Watt FM, Broad S, Prowse DM. Cultivation and retroviral infection of human epidermal keratinocytes. In: Celis JE, ed. Cell biology: a laboratory handbook. New York: Academic Press, 2004.

46 Read J, Watt FM. A model for in vitro studies of epidermal homeostasis: proliferation and involucrin synthesis by cultured human keratinocytes during recovery after stripping off the suprabasal layers. J Invest Dermatol 1988; 90:739-743.

47 Hobbs RM, Watt FM. Regulation of interleukin-1 $\alpha$ expression by integrins and epidermal growth factor receptor in keratinocytes from a mouse model of inflammatory skin disease. $J$ Biol Chem 2003; 278:19798-19807.

48 Ushmorov A, Ratter F, Lehmann V, Dröge W, Schirrmacher V, Umansky V. Nitric oxide-induced apoptosis in human leukemic lines requires mitochondrial lipid degradation and cytochrome c release. Blood 1999; 93:2342-2352.

49 Frye M, Fisher AG, Watt FM. Epidermal stem cells are defined by global histone modifications that are altered by Mycinduced differentiation. PLOS ONE 2007; 2:e763. 